\title{
Water Distribution Networks resilience analysis: a comparison between Graph Theory-based approaches and Global Resilience Analysis
}

Alessandro PAGANO, PhD. Water Research Institute - National Research Council (IRSACNR). alessandro.pagano@ba.irsa.cnr.it ORCID: 0000-0002-2511-9396

Chris SWEETAPPLE, PhD. Centre for Water Systems, College of Engineering, Mathematics and Physical Sciences, University of Exeter, Exeter, EX4 4QF, U.K. ORCID: 0000-00029329-5367

Raziyeh FARMANI, PhD. Centre for Water Systems, College of Engineering, Mathematics and Physical Sciences, University of Exeter, Exeter, EX4 4QF, U.K. ORCID: 0000-00018148-0488

Raffaele GIORDANO, PhD. Water Research Institute - National Research Council (IRSACNR). ORCID: 0000-0002-8103-4055

David BUTLER, PhD. Centre for Water Systems, College of Engineering, Mathematics and Physical Sciences, University of Exeter, Exeter, EX4 4QF, U.K. ORCID: 0000-0001-55153416 


\begin{abstract}
The structure and connectivity of infrastructure systems such as water distribution networks (WDNs) affect their reliability, efficiency and resilience. Suitable techniques are required to understand the potential impacts of system failure(s), which can result from internal (e.g. water hammer) or external (e.g. natural hazards) threats. This paper aims to compare two such techniques: Graph Theory (GT) and Global Resilience Analysis (GRA). These are applied to a real network - L'Aquila (central Italy) - and two benchmark networks - D-Town and EXNET. GT-based metrics focus on the topology of WDNs, while GRA provides a performance-based measure of a system's resilience to a given system failure mode. Both methods provide information on the response of WDNs to pipe failure, but have different data requirements and thus different computational costs and precision. The results show that although GT measures provide considerable insight with respect to global WDN behavior and characteristics, performance-based analyses such as GRA (which provide detailed information on supply failure duration and magnitude) are crucial to better understand the local response of WDNs to pipe failure. Indeed, particularly for complex networks, topological characteristics may not be fully representative of hydraulic performances and pipe failure impacts.
\end{abstract}

Keywords: resilience assessment, water distribution networks, Global Resilience Analysis, Graph-Theory metrics. 


\section{INTRODUCTION}

2 The functioning of water supply infrastructure is crucial for the safety and well-being of communities,

3 but it is threatened by an increasing number of both human actions and natural disasters, which are

4 often unusual, unforeseeable and unavoidable (Meng et al. 2018, Pagano et al. 2018a). Consolidated risk management tools are often of limited use as they are unable to address unpredictable threats. A shift from risk to resilience management is therefore emerging, since a resilient system is capable of coping with unexpected, not-forecasted hazards (for instance, extreme weather events) (Meng et al. 2018).

9 Resilience can be defined as "the degree to which the system minimizes level of service failure magnitude and duration over its design life when subject to exceptional conditions" (Butler et al.

11 2016). A comprehensive resilience assessment of a water distribution network (WDN), therefore, requires knowledge of the level of service failure magnitude and duration when faced with threats.

13 Available approaches for assessing WDN resilience (e.g. Shin et al. 2018) can be broadly classified 14 as either 'property-based' or 'performance-based'.

15 'Property-based' approaches investigate the susceptibility of WDNs to failure, focusing on the link between system performance and inherent structural properties such as robustness, diversity, connectivity and redundancy (Yazdani and Jeffrey 2012, Butler et al. 2016). One such approach considers the WDN as a set of multiple interconnected and interacting nodes (e.g. demand points, tanks and reservoirs) and edges (e.g. pipes, pumps and valves) and uses Graph Theory (GT) to explicitly analyze key properties, thus providing an intuitively robust and quantitative evaluation

21 (Yazdani and Jeffrey 2011). The use of GT-based metrics found wide and early acceptance in WDN research applications (e.g. Jacobs and Goulter 1989, Walski 1993). Many researchers employed such methodologies for reliability analysis (e.g. Ostfeld 2005, Yazdani and Jeffrey 2012) and to investigate failure conditions due to several phenomena (e.g. random failures, deterioration, catastrophic events, targeted attacks). GT may also provide simplified information on system resilience by enabling identification of structural vulnerabilities and points of failure (Yazdani et al. 2013) and analyzing 
1 the disruption caused by the failure of individual components (Yazdani and Jeffrey 2012, Meng et al.

2 2018). Both 'network-level' and 'local' GT metrics are used for such purposes (Yazdani et al. 2011,

3 Yazdani and Jeffrey 2012, Pagano et al. 2018a). The former employs simple graph metrics to analyze

4 global network features. The latter is based on the removal of components (either random or targeted)

5 to assess different failure scenarios (Yazdani et al. 2013). Although some studies have identified a

6 direct correlation between GT metrics and network performance (e.g. Meng et al. 2018), an explicit

7 focus on the role and potentiality of GT representations of pipe networks for WDN hydraulic performance analysis is still lacking (Torres et al. 2016). In fact, whilst specific properties may provide resilient performance, this cannot be guaranteed (Butler et al. 2016). Particularly, the

10 increasing level of complexity and interconnection in water systems is a challenge since any change

11 in the network characteristics has consequences on hydraulic function (Yazdani et al. 2013).

12 'Performance-based' approaches require modelling of performance (i.e. the ability of a network to maintain supply under failure conditions) under multiple system failure scenarios, using hydraulic models. Both single component failure analysis and global resilience analysis (GRA) can be used (Diao et al. 2016). GRA focuses on level of service provision under any possible magnitude of a given system failure mode, irrespective of the threat that may cause this failure (Diao et al. 2016). For example, in a WDN, the effects of any pipe failure magnitude (e.g. number of pipes failed at the same time) on supply could be captured using GRA. This method overcomes the challenge faced in conventional top-down approaches of identifying all the possible threats (e.g. the causes of pipe failure), and instead focuses on the system failure modes as these are easier to identify and

21 characterize (Butler et al. 2016). GRA results in the generation of response curves (system 22 performance in terms of both supply failure magnitude and duration as a function of system failure magnitude), the area under which provides an indication of how resilient the level of service provision is to a given system failure mode. A reduction in the area under the response curve, therefore, represents an increase in resilience. This is a highly flexible approach and has been applied previously 
1 demonstrated that, in addition to providing a performance-based measure of resilience, GRA can be

2 used to identify scenarios that result in the greatest loss of service, therefore acting as a diagnostic

3 framework and aiding the development of interventions (Diao et al. 2016).

4 Table 1 provides a comparative summary of the main characteristics of GRA and GT.

Table 1. Comparison of the key features of GRA and GT

\begin{tabular}{|c|c|c|}
\hline & GRA & GT \\
\hline Type of approach & 'Performance-based' & 'Property-based' \\
\hline Rationale & $\begin{array}{l}\text { Modelling of performance under multiple } \\
\text { system failure scenarios }\end{array}$ & $\begin{array}{l}\begin{array}{l}\text { Analysis of topological network } \\
\text { properties } \\
\text { connectivity, redundancy) }\end{array} \\
\text { (e.g. robustness, }\end{array}$ \\
\hline Information required & Hydraulic model & Topological information \\
\hline Information provided & $\begin{array}{l}\text { Response curves, i.e. } \text { system } \\
\text { performance (supply failure magnitude } \\
\text { and duration) as a function of system } \\
\text { failure magnitude }\end{array}$ & $\begin{array}{l}\text { Degree of interconnectedness, } \\
\text { topological redundancy, identification } \\
\text { of critical components, response to } \\
\text { perturbations }\end{array}$ \\
\hline Main scope/application & $\begin{array}{l}\text { Resilience assessment based on the } \\
\text { characterization of level of service } \\
\text { provision under any possible magnitude } \\
\text { of a given system failure mode }\end{array}$ & $\begin{array}{l}\text { Classification and comparison among } \\
\text { WDNs, identification of structural } \\
\text { vulnerabilities and points of failure, } \\
\text { simplified resilience assessment }\end{array}$ \\
\hline Key advantages & $\begin{array}{l}\text { The potential effects of all threats (even } \\
\text { unknown) that could result in a specific } \\
\text { system failure mode are captured in a } \\
\text { single analysis. }\end{array}$ & $\begin{array}{l}\text { The analysis can be performed without } \\
\text { considering hydraulic information, } \\
\text { although this can be included using } \\
\text { weights if available. }\end{array}$ \\
\hline Potential limitations & $\begin{array}{l}\text { The analysis may be not feasible for big } \\
\text { networks, with a high number of } \\
\text { elements }\end{array}$ & $\begin{array}{l}\text { The results may be not fully } \\
\text { representative for networks } \\
\text { complex hydraulic behavior } \\
\end{array}$ \\
\hline $\begin{array}{l}\text { Data and computational } \\
\text { requirements }\end{array}$ & $\begin{array}{l}\text { More data-dependent } \\
\text { computationally intensive }\end{array}$ & $\begin{array}{l}\text { Less data-dependent } \\
\text { computationally intensive }\end{array}$ \\
\hline
\end{tabular}

The relationship between property- and performance-based measures of resilience has received limited attention to date. No comprehensive studies relate resilience with topological attributes of

9 WDNs, and the appropriateness of topological metrics for resilience assessment is unknown (Meng et al. 2018). These knowledge gaps need to be addressed in order to define effective frameworks for

11 assessing and enhancing resilience in practice.

12 This research aims to provide a greater understanding of the extent to which an assessment framework

13 based only on the characterization of topological and connectivity properties may be a surrogate for 14 more detailed simulation-based models. Three different WDNs (detailed in Section 2) are analyzed 15 for this purpose: L'Aquila (a real network), D-Town (a benchmark) and EXNET (a benchmark). 
1 preliminary classification of the structure of each network (Section 3.1); second, a specific local GT-

2 measure is introduced to evaluate and rank the impact of single pipe failures (Section 3.2). Levels of

3 service failure magnitude and duration resulting from any single pipe failure are then obtained as part

4 of a GRA (Section 3.3) and the pipe rankings compared with those derived from the developed GT-

5 based measure (Section 4). Comparison of the results obtained for three highly different WDNs

6 supports the understanding of specific potentialities and limitations of the use of both approaches,

7 and also provides suggestions for future research (Section 5).

\section{CASE STUDY NETWORKS}

9 In order to provide a detailed comparative analysis of the performances of GRA and GT in different conditions, three networks (illustrated in Figure 1) are analyzed: L'Aquila (a real network), EXNET

11 and D-Town (benchmark networks). Basic hydraulic information, both under ordinary conditions and 12 under 'failure' was derived from EPANET models. Key characteristics of the case study networks 13 are provided below.

a) L'Aquila

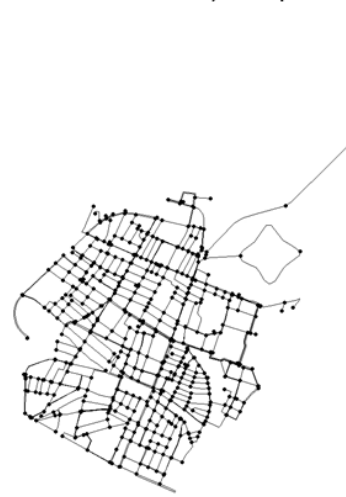

b) D-Town

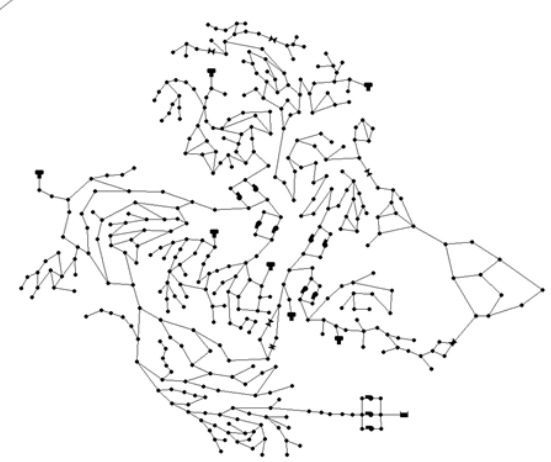

c) EXNET

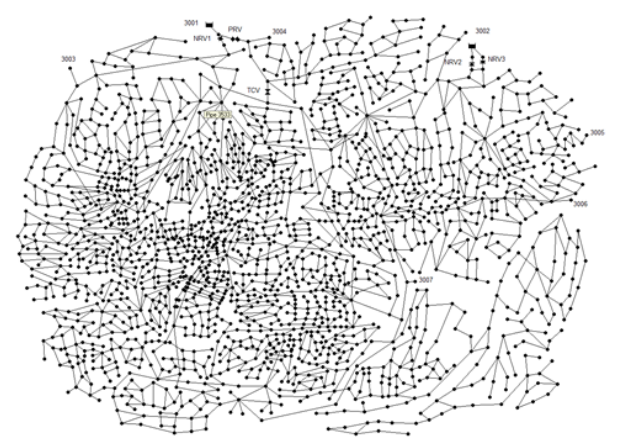

14

Fig. 1 Case study networks

\subsection{L'Aquila}

L'Aquila city (central Italy) was struck by a severe earthquake (6.3 magnitude) on April $6^{\text {th }} 2009$ and the WDN was significantly impacted. Effective resilience assessment methodologies would be of great support to the WDN reconstruction process, since the previous network showed serious 
1 limitations in adaptive capacity (e.g. Pagano et al. 2017, Pagano et al. 2018b). This analysis focuses

2 on the new WDN underlying the historical city center, presented in Figure 1a, which consists of 539

3 junctions, 808 pipes and a single tank (798 $\mathrm{m}$ above sea level, $2000 \mathrm{~m}^{3}$ capacity). As the

4 reconstruction process is still ongoing, significant uncertainties exist over hydraulic operation, and

5 investigation into the potential use of property-based analyses is highly relevant.

$6 \quad 2.2$ D-Town

7 D-Town (Figure 1b) is a benchmark WDN consisting of five district metered areas. In total, it contains 399 junctions, 7 storage tanks, 443 pipes, 11 pumps, 5 valves and a single reservoir. D-Town is highly relevant to this study since it is characterized by complex hydraulic operation, despite the limited

10 number of elements.

\section{$11 \quad 2.3$ EXNET}

12 EXNET (Figure 1c) has been set up by the University of Exeter as a realistic and challenging problem.

13 The network consists of relatively small pipes and few transmission mains, with a large head-loss 14 range at the extremities of the system, making it highly sensitive to demand increases. EXNET 15 contains 1893 junctions (5 of which receive water from adjacent systems), 2462 pipes, 8 valves and 162 reservoirs. This benchmark is of particular interest since it is characterized by complex hydraulic 17 operation and by a high number of elements.

\section{METHODOLOGY}

\subsection{Network-level GT metrics}

21 A graph $G=G(n, m)$ consists of $n$ nodes and $m$ edges. A WDN can be specifically modelled as a graph with nodes/vertices connected by links/edges, and a set of data attributed to them (e.g. nodal demand, edge capacity, flow direction, energy losses) (Meng et al. 2018). The key characteristic of a WDN is that every node should be connected, by at least one path, to one or more source node(s) (e.g.

25 a tank). The structure of a graph could be expressed, mathematically, as an adjacency matrix $A$, i.e. a 
1 node $i$ and node $j$ ). The adjacency matrix is the basis for the calculation of topological metrics.

2 Table 2 identifies a set of network-level GT metrics that are widely used for WDN analysis. They are

3 mathematical attributes related to the main topological properties of networks, which can be related

4 to system resilience (Meng et al. 2018). Using a multi-metric approach, based on multiple attributes,

5 helps identify and compare relevant network properties (Hwang and Landsey 2017).

Table 2 Set of network-level GT metrics used.

\begin{tabular}{|c|c|c|}
\hline Metric & Formula & Description \\
\hline $\begin{array}{l}\text { Average } \\
\text { node degree, } \\
k\end{array}$ & $k=\frac{2 m}{n}$ & $\begin{array}{l}\text { A basic measure of connectivity. It reflects the overall topological } \\
\text { similarity of the network to perfect grids or lattice-like structures } \\
\text { (Yazdani et al. 2011; Yazdani and Jeffrey 2012; Yazdani et al. 2013, } \\
\text { Zeng et al. 2017, Hwang and Linsey, 2017). Higher values suggest higher } \\
\text { redundancy and the existence of multiple paths (Hwang and Linsey } \\
\text { 2017). }\end{array}$ \\
\hline $\begin{array}{l}\text { Average path } \\
\text { length, } \\
\quad l_{T}\end{array}$ & $l_{T}=\frac{1}{n(n-1)} \cdot \sum_{i, j} d\left(v_{i}, v_{j}\right)$ & $\begin{array}{l}\text { The value of the average distance } d \text { along the shortest paths between any } \\
\text { two pairs of nodes }\left(v_{i}, v_{j}\right) \text {, compared to all possible pairs of network nodes } \\
\text { (Yazdani et al. 2011; Yazdani and Jeffrey 2012; Yazdani et al. 2013; } \\
\text { Porse and Lund 2016). }\end{array}$ \\
\hline $\begin{array}{l}\text { Clustering } \\
\text { coefficient, } \\
C_{c}\end{array}$ & $C_{c}=\frac{3 n_{\Delta}}{n_{3}}$ & $\begin{array}{l}\text { Based on the ratio of the number of triangles } n_{\Delta} \text { to the number of } \\
\text { connected triples } n_{3} \text {. It provides a measure of redundancy by quantifying } \\
\text { the density of triangular loops. It is usually smaller in grid-like structures. } \\
\text { Higher values indicate a more clustered network (Yazdani et al. 2011; } \\
\text { Porse and Lund 2016). It describes the tightness of connected } \\
\text { communities (Hwang and Linsey 2017). }\end{array}$ \\
\hline $\begin{array}{l}\text { Critical } \\
\text { breakdown } \\
\text { ratio, } f_{c}\end{array}$ & $f_{c}=\frac{1}{\frac{k^{2}}{k}-1}$ & $\begin{array}{l}\text { Provides a theoretical value for the critical fraction of nodes which need } \\
\text { to be removed for a network to lose its large scale connectivity. The value } \\
\text { thus depends on the average node degree, } k \text { (Yazdani et al. 2011; Yazdani } \\
\text { et al. 2013). }\end{array}$ \\
\hline $\begin{array}{l}\text { Central point } \\
\text { dominance, } \\
\qquad C_{b}\end{array}$ & $C_{b}=\frac{1}{n-1} \sum_{i}\left(b_{v m}-b_{v i}\right)$ & $\begin{array}{l}\text { Measures the concentration of the network topology around a central } \\
\text { location. Its calculation is based on the betweenness centrality of each } \\
\text { network node, } b_{v i} \text {, and of the most central node, } b_{v m} \text {. The value is limited } \\
\text { by the two extremes: } C_{b}=1 \text { for star topology and } C_{b}=0 \text { for regular } \\
\text { networks. (Yazdani et al. } 2011 \text {; Yazdani et al. } 2013 \text {; Porse and Lund } \\
\text { 2016). }\end{array}$ \\
\hline $\begin{array}{l}\text { Density of } \\
\text { bridges, } D_{b r}\end{array}$ & $D_{b r}=\frac{N_{b r}}{m}$ & $\begin{array}{l}\text { Estimates the ratio of the total number of bridges }\left(N_{b r} \text {, i.e. the edges }\right. \\
\text { whose failure may potentially isolate a part of the network) over all edges, } \\
m \text { (Yazdani et al. 2011). }\end{array}$ \\
\hline $\begin{array}{l}\text { Graph } \\
\text { Diameter, } \\
D(G)\end{array}$ & $D(G)=\max \left\{d\left(v_{i} v_{j}\right)\right\}$ & $\begin{array}{l}\text { The maximum geodesic distance between any two nodes. It captures the } \\
\text { maximum eccentricity of nodes in the network and provides a basic } \\
\text { measure of topological and geographical spread of the network (Yazdani } \\
\text { et al. 2011, Torres et al. 2016, Zeng et al. 2017). }\end{array}$ \\
\hline $\begin{array}{l}\text { Link density, } \\
\qquad q\end{array}$ & $q=\frac{2 m}{n(n-1)}$ & $\begin{array}{l}\text { The fraction between the maximum number of possible edges and those } \\
\text { which are actually present (Yazdani et al. 2011, Torres et al. 2016, Zeng } \\
\text { et al. 2017, Hwang and Linsey 2017). A higher } q \text { indicates a more } \\
\text { connected network. }\end{array}$ \\
\hline $\begin{array}{l}\text { Spectral gap, } \\
\Delta \lambda\end{array}$ & $\Delta \lambda$ & $\begin{array}{l}\text { The difference between the first and second eigen values of the adjacency } \\
\text { matrix. A small spectral gap would probably indicate the presence of } \\
\text { articulation points or bridges (Yazdani et al. 2011; Yazdani et al. 2013). }\end{array}$ \\
\hline $\begin{array}{l}\text { Algebraic } \\
\text { connectivity } \\
\lambda_{2}\end{array}$ & $\lambda_{2}$ & $\begin{array}{l}\text { The second smallest eigenvalue of the normalized Laplacian matrix of } \\
\text { the network. It quantifies the network's structural robustness and fault } \\
\text { tolerance. A larger value of algebraic connectivity indicates enhanced } \\
\text { fault tolerance and robustness against efforts to cut the network into }\end{array}$ \\
\hline
\end{tabular}


Meshedness coefficient, $R_{m}$

$$
R_{m}=\frac{m-n+1}{2 n-5}
$$
loops in planar graph. It ranges between 0 for tree-like and 1 for grid-like networks. (Yazdani et al. 2011; Yazdani and Jeffrey 2012; Torres et al. 2016; Porse and Lund 2016). A larger $R_{m}$ corresponds to a more connected network (Hwang and Linsey, 2017).

Physical and operational attributes of nodes and edges can be used to compute network-level GT metrics in a weighted and directed form (Yazdani and Jeffrey 2012, Porse and Lund 2016). For the purposes of this study, network-level metrics are all computed as undirected and unweighted, since WDN operating conditions (e.g. flow direction) may change under failure conditions.

\subsection{Local GT measures for pipe ranking}

The proposed local GT-based analysis framework aims to identify and rank the most crucial elements for system operation in case of failures, relying on topological features only. Specifically, the methodology focuses on potential changes in connection between demand nodes and supply sources caused by single-pipe failures. Since multiple connections often exist between a source and a node, identifying all routes would be computationally expensive. However, limiting the analysis to the 'shortest path' is an appropriate assumption (e.g. Yazdani et al. 2013).

Following Herrera et al. (2016), a surrogate measure of the energy losses is a hydraulically relevant - and easy to quantify - measure of how well a node is connected to the available source(s). Using purely topological characteristics, the energy losses on the edges are proportional to $f \cdot L / D$, where $f$ is the friction factor [-] and $L$ and $D$ are the length [m] and diameter [m] of the edge respectively. The shortest path is thus the one with the lowest value of total energy loss.

The first step of the edge ranking procedure requires identification of the source nodes $(S)$, and computation of the shortest path $(S P)$ from each source to all other nodes of the network $(s)$ under ordinary conditions $\left(S P_{s, i, 0}\right)$. The Dijkstra shortest-paths algorithm is used for this purpose (Dijkstra 1959). Each $S P$ is characterized through a sequence of $K-1$ nodes and $K$ edges and weighted according to the total energy loss (weight $=\sum_{k=1}^{K} f(k) \cdot L_{k} / D_{k}$ ). The second step of the analysis consists of 
1 paths $\left(S P_{s, i, j}\right)$. Comparison between $S P_{s, i, 0}$ and $S P_{s, i, j}$ then allows the change in connectivity between

2 nodes and sources as a consequence of the failure of the edge $j$, to be assessed. Potential scenarios

3 are: 1) the shortest paths do not change; 2) the $S P_{s, i, j}$ returns infinity, meaning that the demand nodes

4 on that path become disconnected from the source $s$, and that the edge $j$ is a bridge; 3 ) the shortest

5 paths between the source $s$ and one or more nodes increase. These three cases are represented

6 graphically in Figure 2.

7 In case (1), the role of edge $j$ in the global operation of the WDN can be considered negligible. In

8 case (2), the total nodal demand that becomes disconnected from all sources once edge $j$ is removed

$9 \quad\left(D D_{j}[1 / \mathrm{s}]\right)$ is computed according to Eq. 10:

$$
D D_{j}=\sum_{s=1}^{S} \sum_{k=1}^{K-1} Q_{k}
$$

10 Edges with a positive $D D_{j}$ value are thus ranked accordingly. Particularly in simple networks with a

11 single source, this analysis may identify specific parts of the network completely cut off from the 12 water supply.

13 In case (3), the impact of edge failure is estimated by computing the shortest path change $\left(S P C_{s, i, j}\right.$,

14 Eq. 11) between all $n$ nodes and $S$ sources, and the cumulate value (Eq. 12):

$$
\begin{aligned}
& S P C_{s, i, j}=\left(S P_{s, i, j}-S P_{s, i, 0}\right) \\
& S P C_{j}=\sum_{S=1}^{S} \sum_{i=1}^{n} S P C_{s, i, j}
\end{aligned}
$$

17 Edges with a positive value of $S P C_{j}$ can be thus ranked accordingly. Two subsets of edges may be 18 therefore identified, and particular attention should be given to those having the highest values of 19 either $D D_{j}$ or $S P C_{j}$. 


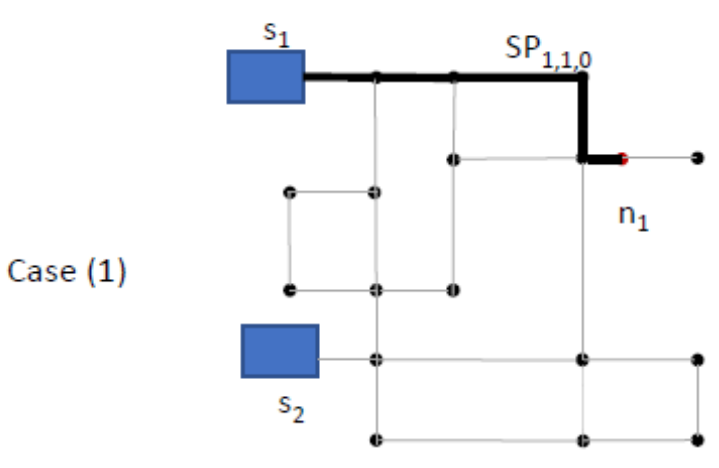

Case (2)

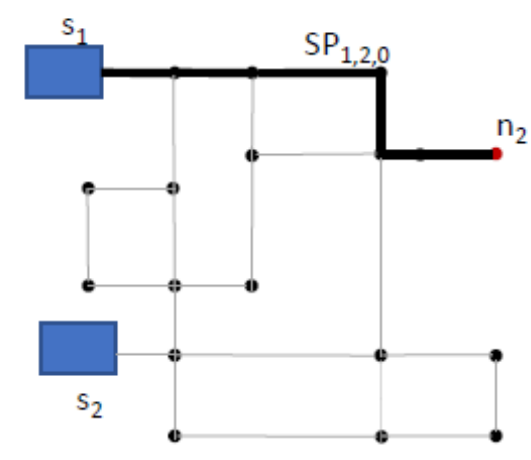

Case (3)

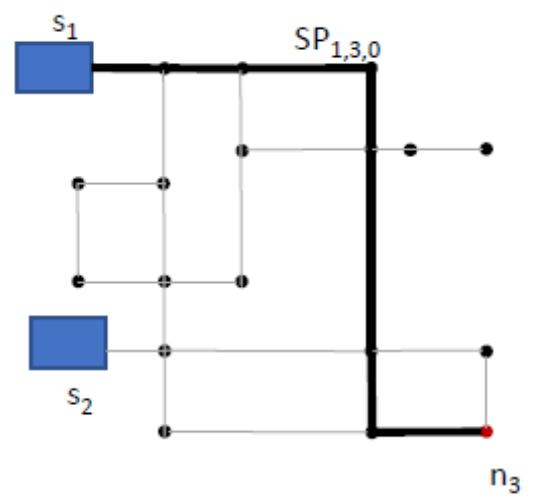

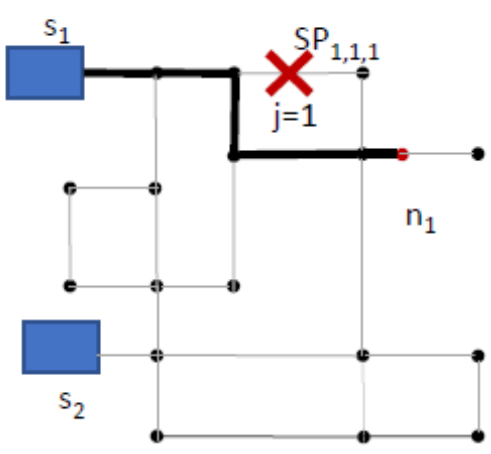
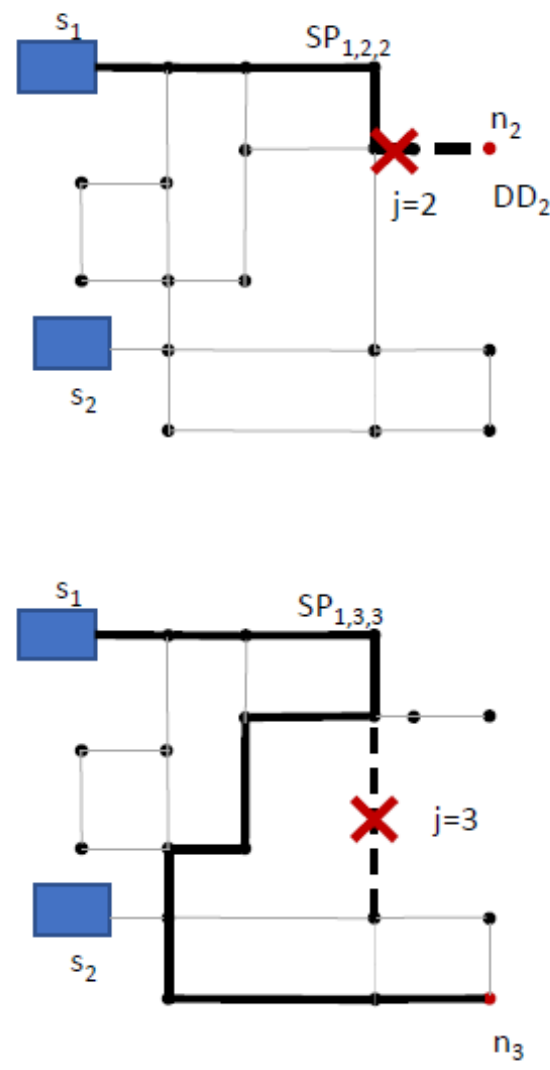

2 Fig. 2. Graphical representation of the potential impact of edge removal on WDN connectivity: case

3 1) no changes occurred in the $S P$ between the source $s_{1}$ and the node 1 after the removal of edge 1;

4 case 2) the removal of the edge 2 results in the disconnection between the source $s_{1}$ and the node 2;

5 case 3 ) the removal of the edge 3 results in the increase of the shortest path between the source $s_{1}$ and

6 the node 3. 


\subsection{Global resilience analysis}

2 A detailed description of the GRA methodology is provided by Diao et al. (2016). Key steps are as 3 follows:

1. Identify the system failure mode(s) for analysis and an appropriate measure of magnitude.

2. Identify the required level(s) of service and appropriate measure(s).

3. Calculate the required level of service measure(s) under every system failure magnitude. Where multiple scenarios are possible for each system failure magnitude (e.g. for failure of $1 \%$ of pipes in the system), sampling and targeted scenario development are used, as detailed by Diao et al. (2016).

4. Plot each level of service measure as a function of system failure magnitude

Further information on the system failure mode, level of services measures and network simulations in this study are given in the following sections.

\subsubsection{System failure mode}

Multiple system failure modes exist; to enable comparison of GRA and GT, this study considers pipe failure. The percentage of pipes in the network failed represents the system failure magnitude and values in the range $0-100 \%$ are evaluated (note that 'magnitude' is used here to address the quantity of pipes failed, not the frequency of pipe failure). Pipe failures are modelled in EPANET by setting the corresponding pipe statuses to 'closed'. They are applied at 10 A.M. so as to capture the effects of peak demand, and maintained to the end of a 24 hour simulation.

Random pipe failure samples are generated at every failure magnitude and, additionally, pipe failure combinations resulting in the minimum and maximum response at each pipe failure magnitude are carried forward for targeted failure scenario development, as described by Diao et al. (2016). This approach is found to provide a good estimation of the minimum, mean and maximum response curves whilst maintaining a manageable computation time.

\subsubsection{Level of service}

Chosen measures of level of service failure are: a) Supply failure duration, and b) Supply failure 
1 magnitude. Given that EPANET is demand driven and supply is not directly calculated, supply at

2 each time step and node is estimated using Eq. 13:

$$
\begin{gathered}
\text { if } P_{j, i} \leq 0 \quad: \quad S_{j, i}=0 \\
\text { if } 0<P_{j, i}<P_{\text {lim }}: \quad S_{j, i}=D_{j, i} \cdot \sqrt{P_{j, i} / P_{l i m}} \\
\text { if } P_{j, i} \geq P_{\text {lim }}: S_{j, i}=D_{j, i}
\end{gathered}
$$

3 Where: $P_{j, i}=$ pressure at node $j$ at time $I[\mathrm{~m}] ; S_{j, i}=$ supply at node $j$ at time $I[1 / \mathrm{s}] ; P_{\text {lim }}=$ required

4 minimum pressure, set to $15 \mathrm{~m}[\mathrm{~m}] ; D_{j, i}=$ demand at node $j$ at time $I[1 / \mathrm{s}]$.

5 Supply failure duration is calculated using Eq. 14; this gives a (unitless) value normalized with respect 6 to the system (pipe) failure duration.

$$
\text { Supply failure duration }=\frac{\sum_{0}^{i=T}\left(F_{i} \cdot t_{i}\right)}{F_{P}}
$$

7 Where: $F_{i}=$ System supply failure state at time step $i\left(0\right.$ if $S_{i}=D_{i}, 1$ if $\left.S_{i}<D_{i}\right)[-] ; F_{P}=$ total pipe 8 failure duration $[\mathrm{hr}]$.

9 Supply failure magnitude is calculated using Eq. 15, which gives the fraction of network demand not

10 supplied during the pressure failure period.

$$
\text { Supply failure magnitude }=\frac{\sum_{0}^{i=T}\left(\sum_{0}^{j=n}\left(D_{j, i}-S_{j, i}\right)\right)}{\sum_{0}^{i=T}\left(\sum_{0}^{j=n} D_{j, i}\right)}
$$

11 Where: $n=$ number of nodes; $T=$ number of time steps; $t_{i}=$ Duration of time step $i$.

\section{RESULTS}

\section{4.1 WDN characterization based on network-level GT metrics}

14 The values of metrics described in the Table 2 are summarized in Table 3 and their significance with 15 respect to network performance is discussed in the present section. As stated previously, these network-level metrics are computed in unweighted and undirected form. 
Table 3 Network-level GT metrics for the case study WDNs

\begin{tabular}{lccc}
\hline GT metrics & L'AQUILA & D-TOWN & EXNET \\
\hline Nodes, $n$ & 539 & 407 & 1893 \\
Sources, $S$ & 1 & 8 & 9 \\
Edges, $m$ & 808 & 459 & 2467 \\
\hline$k$ & 2.998 & 2.256 & 2.606 \\
$q$ & $5.6 \mathrm{E}-03$ & $5.6 \mathrm{E}-03$ & $1.4 \mathrm{E}-03$ \\
$D(G)$ & 26 & 66 & 54 \\
$C_{b}$ & 0.412 & 0.54 & 0.282 \\
$l_{T}$ & 13.45 & 26.38 & 20.61 \\
$f_{c}$ & $50.05 \%$ & $79.65 \%$ & $62.25 \%$ \\
$R_{m}$ & 0.25 & 0.07 & 0.15 \\
$C_{c}$ & 0.041 & 0.019 & 0.04 \\
$N_{b r}$ & 41 & 190 & 490 \\
$D_{b r}$ & 0.05 & 0.41 & 0.20 \\
$\Delta \lambda$ & $2.10 \mathrm{E}-03$ & $6.78 \mathrm{E}-04$ & $1.49 \mathrm{E}-03$ \\
$\lambda_{2}$ & $2.70 \mathrm{E}-03$ & $6.47 \mathrm{E}-04$ & $1.02 \mathrm{E}-03$ \\
\hline
\end{tabular}

3 The average node degree $(k)$ and link density $(q)$ are key structural measures, representative of

4 network connectivity. Higher $k$ and $q$ values suggest higher network connection, and thus a better 5 resistance to failures (e.g. Zeng et al. 2017).

6 Higher values of the central point dominance $\left(C_{b}\right)$ suggest that D-Town and L'Aquila are more 7 centralized networks than EXNET, i.e. they tend to a 'star' topology with a significant concentration 8 around central locations. The values of the clustering coefficient $C_{c}$ suggest that L'Aquila and 9 EXNET are more tightly connected and have better performance in terms of network efficiency and 10 redundancy. The meshedness coefficient $R_{m}$ confirms the higher redundancy of L'Aquila network.

11 The density of bridges $D_{b r}$ denotes the different presence of elements whose removal may isolate 12 parts of the network.

13 Among the investigated networks, D-Town is the smallest (considering $n$ and $m$ ), but has the highest 14 diameter $D(G)$, which suggests a higher spread. This result is also confirmed by the high value of the $15 l_{T}$, which provides a view of network reachability and efficiency in water transport: shorter paths 16 indicate more efficient networks, and systems with shorter water travel time. 
1 The critical breakdown ratio $f_{c}$ indicates topology robustness. Larger values, as for D-Town, might

2 indicate higher resistance to random failures of components and lower vulnerability.

3 Referring to the spectral properties, higher values of the normalized spectral gap $\Delta \lambda$ (as for L'Aquila)

4 indicate a better optimized connectivity layout and a better robustness. The algebraic connectivity $\lambda_{2}$

5 of L'Aquila is also significantly higher, suggesting enhanced fault tolerance and robustness against

6 efforts to bisect the network, and thus to isolate its parts (Zeng et al. 2017). D-Town has the lowest

$7 \quad$ values for both parameters.

\section{$8 \quad 4.2$ Global resilience analysis}

9 The supply failure magnitude and duration response to pipe failure magnitudes of up to $100 \%$ in the

10 case study networks are shown in Figure 3. Figure 3a shows the maximum (solid line), the mean

11 (dashed line) and the minimum (dotted line) pressure failure duration in L'Aquila. The mean supply

12 failure duration increases rapidly as the number of pipes failed increases. When considering the 13 system as a whole, failure of $18.1 \%$ of pipes (equivalent to 146 pipes) will typically result in supply

14 failure during the entire pipe failure period. Supply failure duration responses are only plotted for 15 L'Aquila, as pressures below 15m (and hence supply failures) are present in D-Town and EXNET at 16 all times, irrespective of the number of pipe failures. Note that, due to the large number of pipes in 17 EXNET and the high computational demand of GRA, it is not feasible to evaluate all pipe failure 18 magnitudes for this system. Figures $3 \mathrm{~b}$-d, therefore, show the response to up to 300 simultaneous 19 pipe failures $(12.2 \%)$ in this system. This number of pipe failures results in mean and maximum 20 supply failure magnitudes of $94 \%$ and $100 \%$ respectively, and thus consideration of higher pipe 21 failure magnitudes would yield little further information of interest. Based on the multiple random 22 and targeted pipe failure scenarios evaluated at each pipe failure magnitude, three sets of curves are 23 shown: the minimum (Figure 3b), the mean (Figure 3c) and the maximum (Figure 3d) response. 
a) L'Aquila supply failure duration

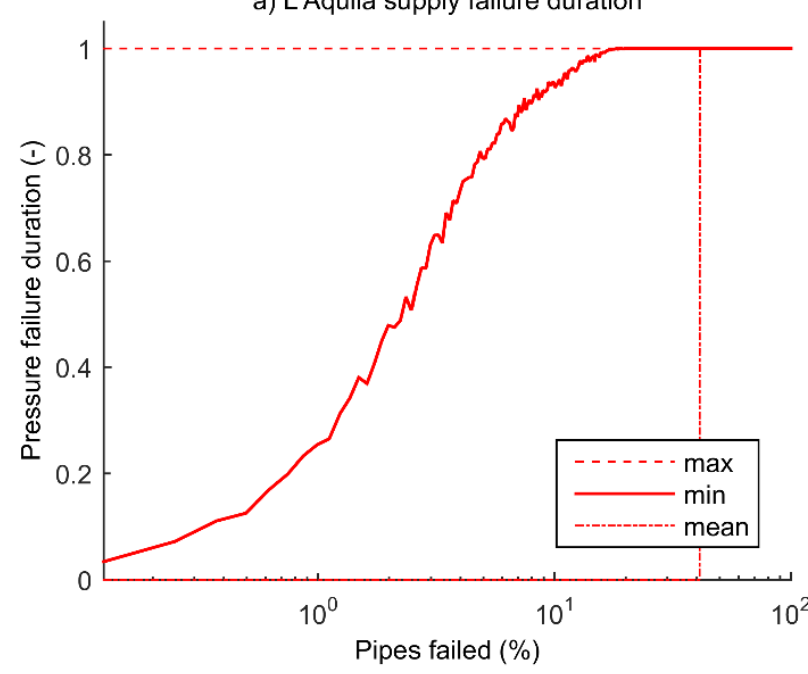

c) Mean supply failure magnitude

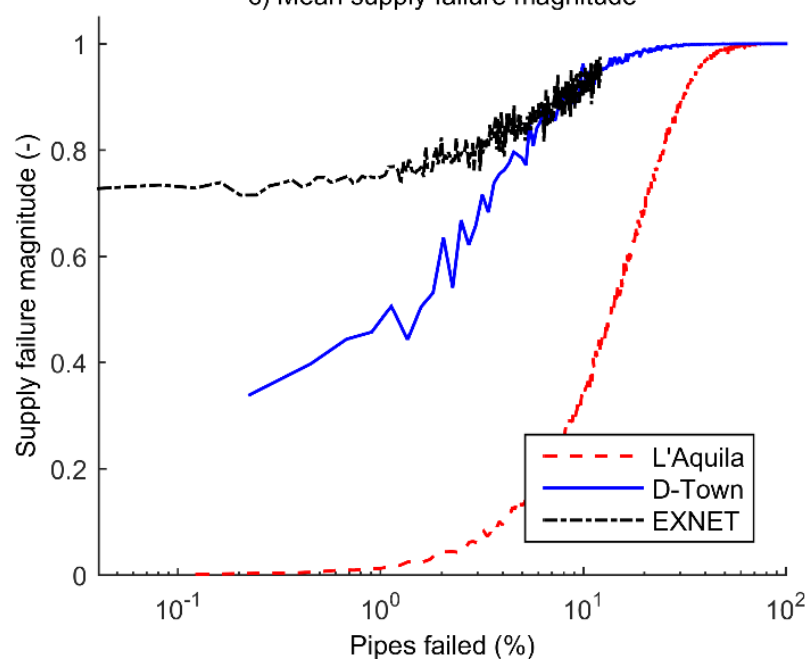

b) Minimum supply failure magnitude

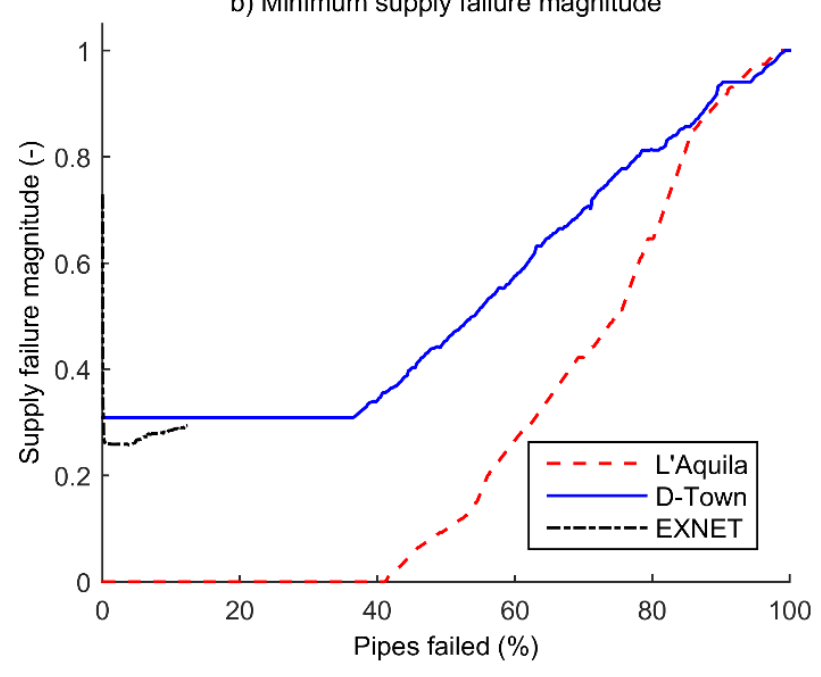

d) Maximum supply failure magnitude

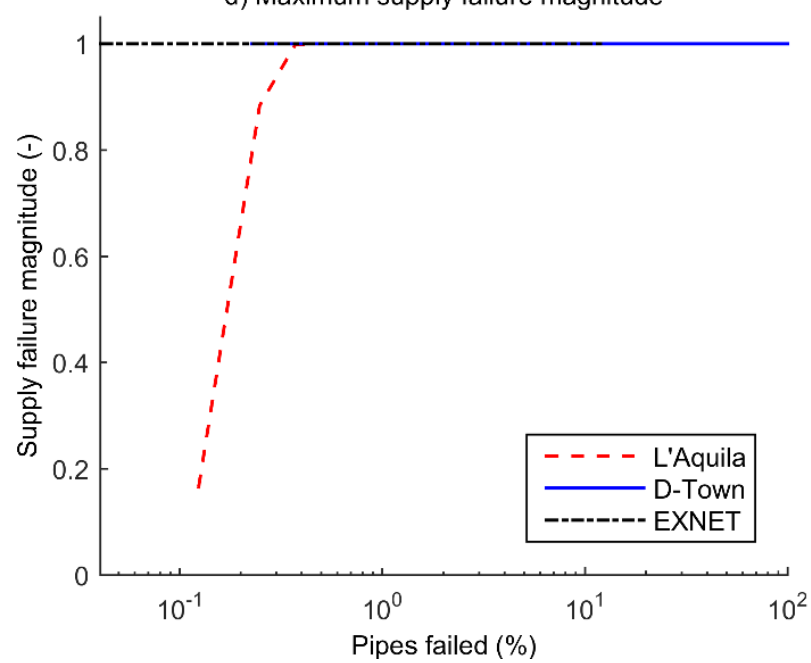

2 Fig. 3 Service failure duration and magnitude response to pipe failure.

4 Analysis of the minimum response curves in Figures 3a and $3 b$ show that the L'Aquila network is 5 capable of maintaining full supply with up to $41.1 \%$ pipe failure (331 pipes). However, the mean 6 supply failure magnitude for this number of pipe failures is $97.5 \%$. On average, at least $99 \%$ of global 7 network demand will be met with up to $0.7 \%$ of pipes failed (i.e. fewer than 7 pipe failures). On average, 7 pipe failures result in a $50.6 \%$ supply failure in D-Town and a $73 \%$ supply failure in

9 EXNET, as these networks have a significant volume of demand affected by unsatisfactory pressure

10 even when no pipe failures are present. The minimum response curve for EXNET, however, does

11 show an initial drop under small pipe failure magnitudes, indicating that there are one or more pipes

12 which, if closed, actually reduce the presence of unsatisfactory pressure in the network. 
1 The maximum supply failure magnitude response curves show that complete loss of supply may occur

2 in L'Aquila with failure of just 4 pipes ( $0.5 \%$ of pipes); however, this can occur in D-Town and

3 EXNET with a single pipe failure. A summary of the mean and maximum supply failure magnitude

4 responses to up to 4 simultaneous pipe failures in the three case study WDNs is given in Table 4.

5 These results suggest that L'Aquila is the most resilient of the three case studies (with respect to pipe

6 failure). To aid identification of critical components and reveal potential focus areas for further

7 improvement, specific pipe failures which result in the maximum supply losses are identified.

8 Table 4 Summary of supply failure magnitudes (percentage of network demand during pressure

9 failure period not supplied) resulting from up to four simultaneous pipe failures and identification of

10 pipes resulting in maximum supply failure magnitude

\begin{tabular}{|c|c|c|c|c|c|c|c|c|c|}
\hline \multirow{2}{*}{$\begin{array}{c}\text { Number } \\
\text { of pipes } \\
\text { failed }\end{array}$} & \multicolumn{3}{|c|}{ L-Aquila } & \multicolumn{3}{|c|}{ D-Town } & \multicolumn{3}{|c|}{ EXNET } \\
\hline & Mean & Max & Pipe ID(s) & Mean & $\operatorname{Max}$ & Pipe ID(s) & Mean & Max & Pipe ID(s) \\
\hline 1 & 0.1 & 16.6 & 902 & 33.9 & 100.0 & P310 or P316 & 72.8 & 100.0 & 3244 \\
\hline 2 & 0.4 & 88.0 & 281,477 & 39.7 & 100.0 & $*$ & 73.4 & 100.0 & $*$ \\
\hline 3 & 0.4 & 99.8 & $281,477,770$ & 44.4 & 100.0 & $*$ & 73.9 & 100.0 & $*$ \\
\hline 4 & 0.6 & 100.0 & $*$ & 45.7 & 100.0 & $*$ & 71.5 & 100.0 & $*$ \\
\hline
\end{tabular}

\subsection{Comparison between GRA and local GT-based measures based on pipe rankings}

13 This section compares the supply failure magnitude response to single pipe failure scenarios, derived 14 as part of the GRA process, with rankings obtained from the proposed local GT-based measure. The 15 aim is to provide an understanding of the extent to which WDN resilience to pipe failure can be estimated based on topological and connectivity characteristics only, given the assumption that EPANET provides an accurate measure of hydraulic performance under single pipe failure conditions in the GRA. In order to limit the analysis to the most relevant elements, only the top-ranked pipes are taken into account. L'Aquila represents the simplest network in terms of hydraulic operation and identification of bridges 
1 is crucial for this kind of network, since their failure could cause the disconnection of wide areas from

2 the water source. The results of single pipe failure scenarios in the GRA suggest that, individually,

3 only 28 (out of 808) pipes have an impact on network performance if they fail individually. The GT

4 analysis suggests that all these pipes are bridges for the WDN. Table S1 in the Supplemental Data

5 lists the highest ranked pipes, based on $D D_{j}$ value and supply failure magnitude resulting from their

6 individual failure (derived from GRA).

7 The results summarized in Table S1 show that the top 28 pipes as identified by GT are all ranked in the top 28 in the performance-based analysis as well. Only one minor difference is present in the ranking, which is a remarkably good result. The performance assessment in case of failure is instead

10 conditioned by the hydraulic regime, which is explicitly included in GRA. The relevance of topology 11 and connectivity to the performance of L'Aquila WDN is particularly high due to the simple structure 12 of the network (i.e. it is supplied by a single source and characterized by a regular, grid-like structure). 13 Therefore, for this network, the GT-based analysis is highly representative of the actual network 14 operation.

15 Both D-Town and EXNET have a complex structure characterized by multiple sources which affect 16 hydraulic operation. EXNET, in particular, is a highly complex WDN and provides a demanding test 17 for the proposed GT-based approach. In both cases, the consistency of results obtained from the two methodologies was assessed by focusing on pipes ranked in the top $10 \%$ according to GRA, and checking how many edges were also identified in the top $10 \%$ when ranked by the local GT-based measures.

21 Focusing on D-Town, the methodology provides remarkably good results, as shown in the Table S2 22 of the Supplementary Material. 95\% of the pipes ranked in the top 10\% according to GRA (38 out of 2340 ), for example, are also in the top $10 \%$ according to the GT-based measures. More specifically, the 24 top 10 ranked pipes according to the values of both $D D_{j}$ and $S P C_{j}$ (highlighted in grey in the Table 25 S2) fall within the top 40 as identified by GRA. 
1 high-ranked pipes according to GRA are in the top $10 \%$ of pipes according to the GT-based rank.

2 Going further into details, only $50 \%$ of the top 10 ranked pipes according to the local GT-based

3 measures (in grey in the Table S3) fall within the top $10 \%$ of pipes according to GRA ranking. Full

4 results for all WDNs, are provided in the Supplementary Information.

5 In order to understand the rationale behind such discrepancies, two EXNET pipe failure scenarios

6 with the greatest difference between their rankings were identified and their hydraulic behavior

7 analyzed. These pipes are physically close to each other and connected at one node: Pipe $3048(\mathrm{D}=$

$81073 \mathrm{~mm})$ and Pipe $3474(\mathrm{D}=900 \mathrm{~mm})$. The location of these pipes is shown in Figure 4. Both pipes

9 are high-ranked according to the GT approach (ranks 12 and 15 respectively based on SPC), whereas

10 only one (pipe 3474) is high-ranked according to GRA and the other (pipe 3048) is among the lowest

11 ranked (ranks 3 and 2462 respectively). The hydraulic operation of the system was investigated

12 considering the impact of single pipe failure on system operation, as shown in Figure 4, which

13 includes: a) identification of the nodes with unsatisfactory pressure due to the failure of pipe 3474 ;

14 b) identification of the nodes with unsatisfactory pressure due to the failure of pipe 3048; c) the flow

15 rate in pipes connected to 3474 after its failure; d) the flow rate in pipes connected to 3048 after its 16 failure. 
a) Failure of edge 3474 ; nodes with pressure failure are shaded

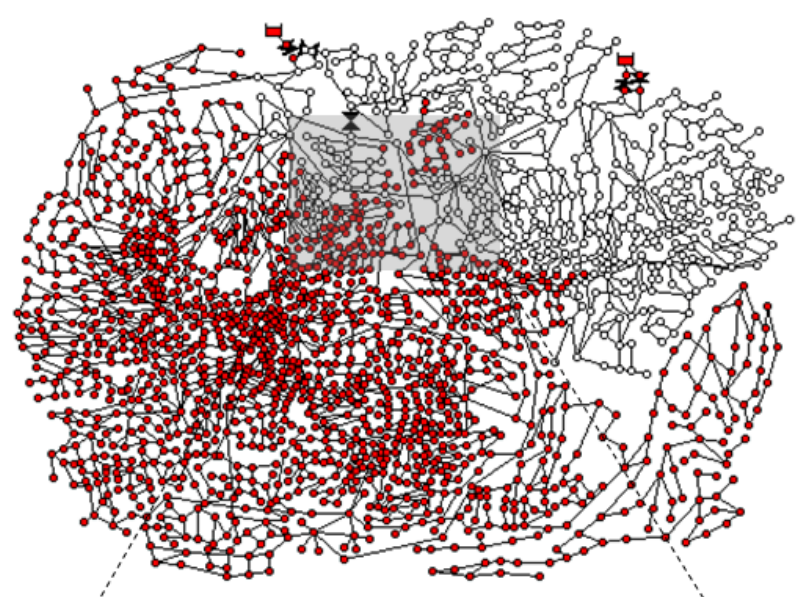

c) Failure of edge 3474 ; line weight proportional to flow rate through pipes

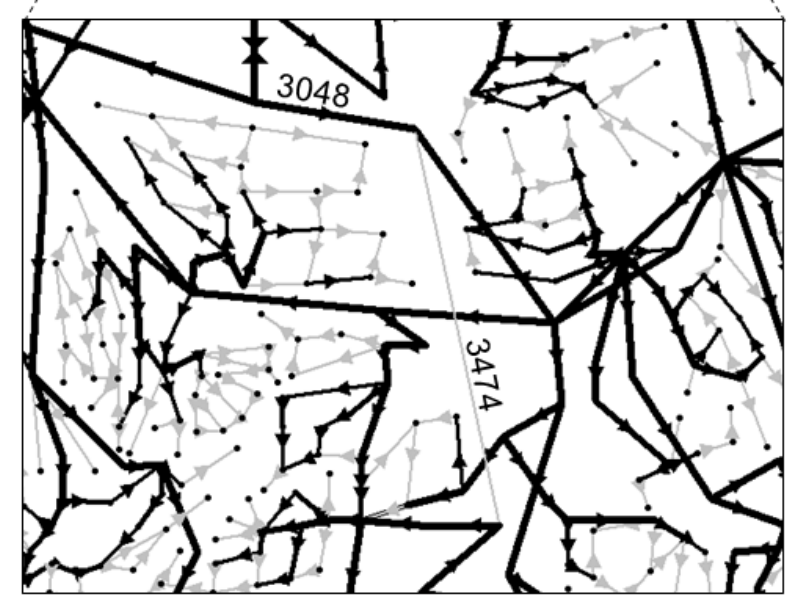

b) Failure of edge 3048; nodes with pressure failure are shaded

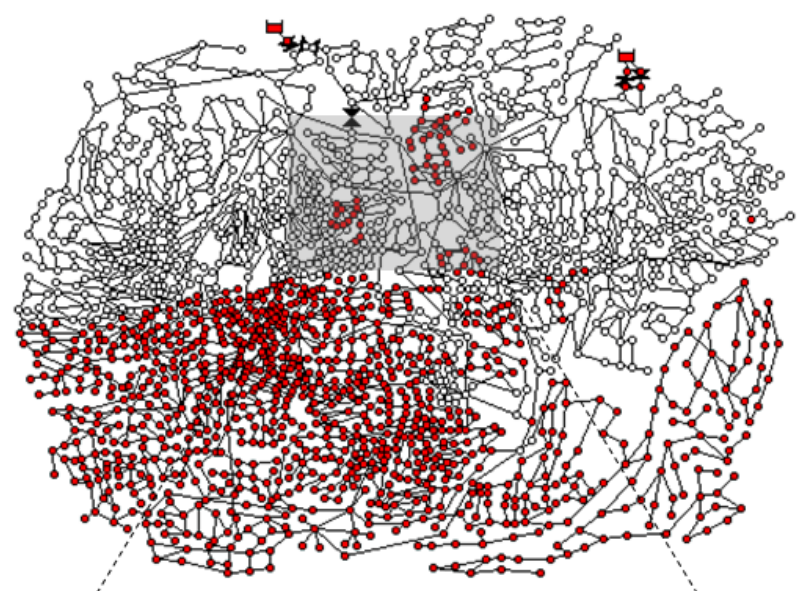

d) Failure of edge 3048; line weight proportional to flow rate through pipes

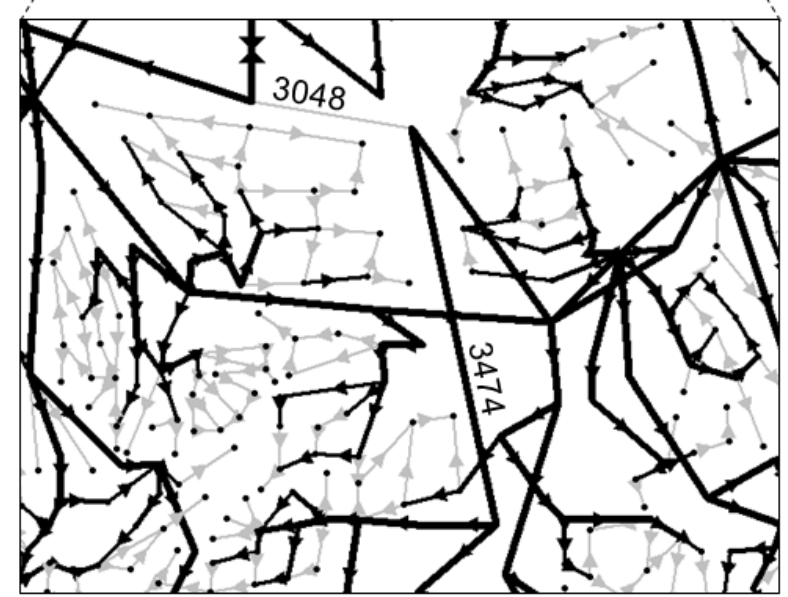

Fig. 4 Comparative analysis of the impact of single pipe failure of edges 3474 and 3048.

Based on the pressures and flow rates shown in Figure 4, it can be seen that the impact of pipe 3474 or 3048 failing individually is highly different, mainly due to the role of pipe 3367: analysis of ordinary operation and failure conditions suggests that when pipe 3048 fails, pipe 3367 is subjected to a change in the flow direction which supports the operation of pipe 3474 . This means that the impact of pipe failure can be partially absorbed by the system, which is resilient enough to adapt to a

9 change in hydraulic conditions. When pipe 3474 fails, pipe 3367 does not support system adaptation, and this results in a wider area of the WDN with pressure below an acceptable value. 


\section{DISCUSSION AND CONCLUSIONS}

2 Both performance- and property- based approaches are used for investigating the behavior of WDNs and supporting resilience assessment, but no comprehensive comparative analysis has previously been performed. In particular, additional efforts are needed in order to support a deeper understanding of their limits and potential, thus facilitating the selection of the most suitable one, considering both the purpose of the analysis to be performed and the WDN characteristics (Shin et al. 2018). This paper presents a critical comparison between two different methodologies belonging to the aforementioned categories, i.e GRA and GT-based metrics. GRA can be used as comprehensive diagnostic framework linking system attributes (e.g. connectivity and capacity) to performance (e.g. level of service), and can be adopted to illustrate the complex dynamic responses of systems to various failure modes. GTbased approaches are highly relevant with a twofold perspective: (1) to propose a 'network-level' classification of different WDNs and provide a better understanding of the influence of key properties (e.g. connectivity, robustness, redundancy) on system resilience, with a relatively fast and inexpensive computation; (2) with the implementation of specific 'local' measures, to determine a pipe ranking defining the impact of single pipe failure on system connectivity.

16 The comparison of results based on the local GT-based measures and GRA for three highly different 17 WDNs enables conclusions to be drawn regarding the potential and applicability of these methodologies for resilience assessment also in other networks. Firstly, network-level topological and connectivity aspects are certainly useful to characterize a WDN, since the interconnectedness of the system is relevant for its operation both in ordinary conditions and under failure. Particularly, the 21 selection of a set of network-level GT-based metrics could be highly useful in order to describe network-level system structure and characteristics. Network-level topological properties can be useful as surrogate measures of global system resilience. Secondly, a deeper understanding and modeling of WDN response to stress requires the development and computation of local GT metrics, which explicitly account for the connectivity of the system with water sources, along with the role that single pipes might have on system operation. Indeed, a comprehensive metric of the impact of 
1 pipe failure in terms of network connectivity should take into account both the possibility of isolation

2 for specific parts of the network, and the increase of the shortest paths between source(s) and demand nodes, which may cause a substantial reduction of pressure. Nevertheless, the appropriateness and

4 effectiveness of such methods may vary significantly with network complexity and according to the 5 specific operating conditions, and additional research is needed in this direction. The analyses

6 summarized in the present paper suggest that the effectiveness and reliability of GT-based metrics is 7 significantly higher for WDNs with a basic structure (e.g. single source, regular structure, limited size) and simple operating conditions. In such cases (e.g. L'Aquila), GT could support the effective preliminary identification of the most critical pipes, thus helping to avoid the computational effort associated with other methods. For more complex networks (e.g. EXNET), the topology of the

11 network is only partially representative of system operation, since the hydraulic conditions may 12 significantly change as a consequence of pipe failure and result in local effects which are hard to predict without hydraulic modelling. Additional efforts are needed to support a more effective and

14 reliable implementation of property-based approaches in case of failure (Hwang and Lansey 2017, 15 Shin et al. 2018). As the GRA implementation suggests, evaluation of hydraulic system performance 16 is essential for comprehensive resilience analysis of complex WDNs and can provide additional 17 information with respect to the effects of multiple pipe failures. Future research activities should be 18 also oriented towards a comprehensive comparison of multiple different resilience assessment 19 measures in a wider set of WDNs.

\section{ACKNOWLEDGEMENTS}

21 The authors would like to thank Gran Sasso Acqua S.p.A. for their support in the analysis of L'Aquila WDN, and for sharing data and information. The GRA work forms part of a 5-year fellowship for the last author funded by the UK Engineering \& Physical Sciences Research Council (EP/K006924/1). 


\section{Supplementary Information. Water Distribution Networks}

3 resilience analysis: a comparison between Graph Theory-based

4 approaches and Global Resilience Analysis

5 The following Tables include full details on the comparison performed on the analyzed networks

6 using both GT-based local measures and GRA. Concerning L'Aquila WDN the comparison is limited

7 to the 28 pipes which are high-ranked according to GRA. For D-Town and EXNET, instead, the

8 comparison is focused on the top $10 \%$ of high-ranked pipes according to GRA ('GRA rank').

9 As far as the GT rank is concerned, it is based on either $D D_{j}$ or $S P C_{j}$ values ('GT rank $-D D_{j}$ ' and

10 'GT rank $-S P C_{j}^{\prime}$ '), in case the effect of edge removal is a disconnection of demand node(s) or an

11 increase in the shortest path between source and nodes, respectively. No values are reported in case

12 the removal of the edge has no effects on the shortest paths between the source and the nodes. The

13 top 10 pipes according to both GT-based measures are highlighted in grey.

Table S1. Comparison of pipe rankings derived from GT and GRA for L'Aquila Case.

\begin{tabular}{|c|c|c|c|}
\hline \multirow{2}{*}{ Pipe ID } & \multirow{2}{*}{ GRA rank } & \multicolumn{2}{|c|}{ GT rank } \\
\hline & & $D D_{j}$ & $S P C_{j}$ \\
\hline 902 & 1 & 1 & \\
\hline 579 & 2 & 2 & \\
\hline 569 & 3 & 3 & \\
\hline 1063 & 4 & 4 & \\
\hline 992 & 5 & 5 & \\
\hline 1041 & 6 & 6 & \\
\hline 646 & 7 & 7 & \\
\hline 512 & 8 & 8 & \\
\hline 695 & 9 & 9 & \\
\hline 923 & 10 & 10 & \\
\hline 484 & 11 & 11 & \\
\hline 66 & 12 & 12 & \\
\hline 911 & 13 & 13 & \\
\hline 67 & 14 & 15 & \\
\hline 73 & 15 & 16 & \\
\hline 421 & 16 & 17 & \\
\hline 473 & 17 & 18 & \\
\hline 901 & 18 & 19 & \\
\hline 502 & 19 & 14 & \\
\hline 13 & 20 & 20 & \\
\hline 826 & 21 & 21 & \\
\hline
\end{tabular}




\begin{tabular}{ccc}
\hline 184 & 22 & 22 \\
\hline 481 & 23 & 23 \\
\hline 24 & 24 & 24 \\
\hline 107 & 25 & 25 \\
\hline 108 & 26 & 26 \\
\hline 109 & 27 & 27 \\
\hline 436 & 28 & 28 \\
\hline
\end{tabular}


1 Table S2. Comparison of pipe rankings derived from GT and GRA for D-Town

\begin{tabular}{|c|c|c|c|}
\hline \multirow{2}{*}{ Pipe ID } & \multirow{2}{*}{ GRA rank } & \multicolumn{2}{|c|}{ GT rank } \\
\hline & & $D D_{j}$ & $S P C_{j}$ \\
\hline P310 & 1 & 18 & \\
\hline P316 & 2 & 19 & \\
\hline P98 & 3 & 17 & \\
\hline $\mathbf{P 8 3}$ & 4 & 15 & \\
\hline P97 & 5 & 16 & \\
\hline P22 & 6 & 13 & \\
\hline P100 & 7 & 14 & \\
\hline $\mathbf{P 2 3}$ & 8 & 12 & \\
\hline P25 & 9 & 10 & \\
\hline P34 & 10 & 9 & \\
\hline P102 & 11 & 8 & \\
\hline P24 & 12 & & 26 \\
\hline P110 & 13 & & 24 \\
\hline P99 & 14 & & 19 \\
\hline P17 & 15 & & 21 \\
\hline P18 & 16 & & 20 \\
\hline P19 & 17 & 1 & \\
\hline P20 & 18 & 18 & \\
\hline P468 & 19 & 11 & \\
\hline P297 & 20 & & 17 \\
\hline P21 & 21 & & 39 \\
\hline P892 & 22 & 2 & \\
\hline P96 & 23 & 3 & \\
\hline P467 & 24 & 5 & \\
\hline P445 & 25 & 4 & \\
\hline P465 & 26 & & \\
\hline P237 & 27 & & 2 \\
\hline P379 & 28 & & 1 \\
\hline P308 & 29 & & 7 \\
\hline P256 & 30 & & 9 \\
\hline P252 & 31 & & 8 \\
\hline P238 & 32 & & 3 \\
\hline P292 & 33 & & 4 \\
\hline P933 & 34 & & 10 \\
\hline P934 & 35 & & 11 \\
\hline P293 & 36 & & 5 \\
\hline P996 & 37 & 6 & \\
\hline P291 & 38 & & 6 \\
\hline P397 & 39 & 7 & \\
\hline P319 & 40 & & \\
\hline
\end{tabular}


3 Table S3 Comparison of pipe rankings derived from GT and GRA for EXNET

\begin{tabular}{|c|c|c|c|}
\hline \multirow{2}{*}{ Pipe ID } & \multirow{2}{*}{ GRA rank } & \multicolumn{2}{|c|}{ GT rank } \\
\hline & & $D D_{j}$ & $S P C_{j}$ \\
\hline 3244 & 1 & & \\
\hline 2369 & 2 & & 8 \\
\hline 3474 & 3 & & 15 \\
\hline 3860 & 4 & & 221 \\
\hline 3487 & 5 & & 17 \\
\hline 2381 & 6 & & 16 \\
\hline 3434 & 7 & & 18 \\
\hline 2104 & 8 & & 239 \\
\hline 5257 & 9 & & 42 \\
\hline 3814 & 10 & & 205 \\
\hline 3982 & 11 & & 177 \\
\hline 3939 & 12 & & 187 \\
\hline 2512 & 13 & & 131 \\
\hline 5066 & 14 & & 112 \\
\hline 2096 & 15 & & 42 \\
\hline 5165 & 16 & & 37 \\
\hline 3473 & 16 & & 38 \\
\hline 3419 & 18 & & 209 \\
\hline 5162 & 19 & & 50 \\
\hline 3847 & 20 & 2 & \\
\hline 2087 & 21 & & 132 \\
\hline 3338 & 22 & & \\
\hline 3967 & 23 & & 28 \\
\hline 3490 & 24 & & 113 \\
\hline 5034 & 24 & & 117 \\
\hline 2077 & 26 & & 219 \\
\hline 5221 & 27 & 7 & \\
\hline 4908 & 28 & & 81 \\
\hline 4154 & 29 & & 66 \\
\hline 3924 & 30 & & \\
\hline 2282 & 31 & & 41 \\
\hline 2320 & 32 & & \\
\hline 3353 & 33 & & \\
\hline 4168 & 34 & & 88 \\
\hline 2357 & 35 & & 20 \\
\hline 3538 & 36 & & 96 \\
\hline 3958 & 37 & & 21 \\
\hline 3988 & 38 & & \\
\hline 3273 & 39 & & 40 \\
\hline 2719 & 40 & & 13 \\
\hline 2790 & 41 & & 14 \\
\hline
\end{tabular}




\begin{tabular}{|c|c|c|}
\hline 2667 & 42 & 55 \\
\hline 2139 & 42 & 56 \\
\hline 2456 & 44 & 31 \\
\hline 3019 & 44 & 32 \\
\hline 3339 & 44 & 29 \\
\hline 2073 & 44 & 30 \\
\hline 2292 & 48 & \\
\hline 5063 & 49 & 49 \\
\hline 4070 & 50 & 212 \\
\hline 3740 & 51 & \\
\hline 2599 & 52 & 125 \\
\hline 3528 & 53 & 102 \\
\hline 5212 & 54 & 185 \\
\hline 2546 & 55 & 136 \\
\hline 3547 & 56 & 105 \\
\hline 2176 & 57 & 107 \\
\hline 4004 & 58 & \\
\hline 3046 & 59 & 82 \\
\hline 2451 & 60 & 74 \\
\hline 3713 & 61 & \\
\hline 2355 & 62 & 216 \\
\hline 3418 & 63 & 71 \\
\hline 3047 & 64 & 61 \\
\hline 3422 & 65 & \\
\hline 2619 & 66 & \\
\hline 3438 & 67 & 94 \\
\hline 3783 & 68 & 147 \\
\hline 2297 & 69 & 171 \\
\hline 4173 & 70 & \\
\hline 3249 & 71 & 4 \\
\hline 3379 & 72 & 5 \\
\hline 5132 & 73 & 6 \\
\hline 3269 & 74 & \\
\hline 4174 & 74 & \\
\hline 3274 & 76 & 9 \\
\hline 4186 & 77 & \\
\hline 2380 & 78 & \\
\hline 3716 & 79 & \\
\hline 4854 & 80 & \\
\hline 2465 & 81 & \\
\hline 5161 & 82 & 22 \\
\hline 3443 & 83 & \\
\hline 2393 & 84 & \\
\hline 2454 & 85 & \\
\hline
\end{tabular}




\begin{tabular}{|c|c|c|c|}
\hline 5041 & 86 & & 23 \\
\hline 3410 & 87 & & \\
\hline 4141 & 88 & & 191 \\
\hline 4165 & 88 & & 167 \\
\hline 5047 & 90 & 1 & \\
\hline 3026 & 91 & & \\
\hline 2509 & 92 & & \\
\hline 3917 & 93 & & 36 \\
\hline 2319 & 94 & & \\
\hline 2231 & 95 & & \\
\hline 3307 & 96 & & 215 \\
\hline 2419 & 97 & & \\
\hline 3970 & 98 & & \\
\hline 3090 & 99 & & 118 \\
\hline 5237 & 100 & & \\
\hline 4052 & 101 & & \\
\hline 4077 & 102 & 30 & \\
\hline 2122 & 103 & 29 & \\
\hline 4072 & 104 & & 44 \\
\hline 4060 & 105 & & 46 \\
\hline 2927 & 106 & & 138 \\
\hline 3920 & 107 & & 51 \\
\hline 2407 & 108 & & 48 \\
\hline 4115 & 109 & & 148 \\
\hline 3499 & 110 & & 123 \\
\hline 5120 & 111 & & 43 \\
\hline 3937 & 112 & & 52 \\
\hline 3494 & 113 & 35 & \\
\hline 5153 & 113 & 36 & \\
\hline 3476 & 115 & 37 & \\
\hline 3452 & 115 & 38 & \\
\hline 3500 & 115 & 39 & \\
\hline 2348 & 118 & 40 & \\
\hline 2397 & 118 & 41 & \\
\hline 3449 & 120 & 44 & \\
\hline 2116 & 121 & & 62 \\
\hline 3593 & 121 & & 59 \\
\hline 5102 & 123 & & \\
\hline 3388 & 123 & & \\
\hline 4132 & 125 & & 24 \\
\hline 2285 & 126 & & 149 \\
\hline 2462 & 126 & & 165 \\
\hline 2111 & 128 & & 245 \\
\hline 3643 & 129 & & \\
\hline
\end{tabular}




\begin{tabular}{|c|c|c|c|}
\hline 2651 & 130 & & \\
\hline 3823 & 131 & & 45 \\
\hline 3689 & 132 & & \\
\hline 4057 & 133 & & \\
\hline 3055 & 133 & & \\
\hline 5243 & 135 & & \\
\hline 5195 & 136 & & \\
\hline 3253 & 136 & & \\
\hline 4003 & 138 & & \\
\hline 5199 & 139 & & \\
\hline 5009 & 140 & & \\
\hline 2100 & 141 & & \\
\hline 3993 & 141 & & \\
\hline 3393 & 143 & & \\
\hline 3389 & 144 & 58 & \\
\hline 2557 & 145 & & \\
\hline 5220 & 146 & 49 & \\
\hline 2300 & 146 & 50 & \\
\hline 2579 & 148 & & \\
\hline 5159 & 149 & & 109 \\
\hline 2418 & 150 & & 180 \\
\hline 2406 & 151 & 61 & \\
\hline 3372 & 151 & 62 & \\
\hline 4011 & 153 & 51 & \\
\hline 3639 & 154 & & \\
\hline 2409 & 155 & & 240 \\
\hline 3457 & 156 & & \\
\hline 3439 & 157 & & 137 \\
\hline 3411 & 157 & & 176 \\
\hline 3792 & 159 & & 54 \\
\hline 3625 & 160 & & 114 \\
\hline 3049 & 161 & & \\
\hline 2331 & 161 & & \\
\hline 5177 & 163 & & \\
\hline 2809 & 164 & & 63 \\
\hline 3682 & 165 & & 122 \\
\hline 2948 & 166 & & \\
\hline 2264 & 167 & & 160 \\
\hline 3357 & 168 & 8 & \\
\hline 2759 & 169 & & 65 \\
\hline 2939 & 170 & 64 & \\
\hline 2674 & 171 & & \\
\hline 2466 & 172 & & 119 \\
\hline 2394 & 172 & & 144 \\
\hline
\end{tabular}




\begin{tabular}{|c|c|c|c|}
\hline 2368 & 174 & & \\
\hline 2051 & 175 & & \\
\hline 2124 & 176 & & 120 \\
\hline 4076 & 177 & & \\
\hline 2427 & 178 & 66 & \\
\hline 2410 & 179 & & \\
\hline 3974 & 180 & & \\
\hline 3782 & 181 & & \\
\hline 3446 & 182 & & 233 \\
\hline 2260 & 183 & & \\
\hline 4088 & 184 & & \\
\hline 2539 & 185 & & 228 \\
\hline 2220 & 186 & & 201 \\
\hline 2171 & 187 & & 193 \\
\hline 3692 & 188 & & 70 \\
\hline 2430 & 189 & & 226 \\
\hline 2365 & 190 & & \\
\hline 2201 & 191 & & 225 \\
\hline 2986 & 192 & & 133 \\
\hline 5215 & 192 & & 146 \\
\hline 3849 & 194 & & \\
\hline 3871 & 194 & & \\
\hline 4036 & 196 & & \\
\hline 4008 & 197 & & \\
\hline 5198 & 198 & & \\
\hline 3834 & 199 & & 72 \\
\hline 5261 & 200 & & \\
\hline 3876 & 201 & & \\
\hline 4847 & 202 & & 161 \\
\hline 4878 & 202 & & 140 \\
\hline 4018 & 204 & & \\
\hline 3414 & 205 & & \\
\hline 2389 & 206 & & \\
\hline 2963 & 207 & & 76 \\
\hline 2311 & 208 & & \\
\hline 3798 & 209 & 73 & \\
\hline 4041 & 210 & & \\
\hline 4043 & 211 & & \\
\hline 3004 & 212 & & \\
\hline 2591 & 213 & & \\
\hline 2681 & 214 & & 168 \\
\hline 2995 & 215 & & \\
\hline 5074 & 216 & & \\
\hline 3981 & 217 & & \\
\hline
\end{tabular}




\begin{tabular}{|c|c|c|c|}
\hline 3995 & 218 & 23 & \\
\hline 5076 & 219 & & \\
\hline 3636 & 220 & & \\
\hline 2617 & 221 & & \\
\hline 2714 & 222 & & \\
\hline 4050 & 223 & & 87 \\
\hline 2677 & 223 & & 93 \\
\hline 3317 & 225 & & \\
\hline 2473 & 226 & & 204 \\
\hline 3938 & 227 & & \\
\hline 5145 & 228 & 78 & \\
\hline 4144 & 229 & & \\
\hline 2938 & 230 & & 86 \\
\hline 3909 & 231 & & \\
\hline 5239 & 232 & & 11 \\
\hline 3712 & 232 & & 10 \\
\hline 3690 & 234 & & 77 \\
\hline 2426 & 235 & 26 & \\
\hline 5085 & 236 & & \\
\hline 3390 & 237 & & \\
\hline 5072 & 238 & & 73 \\
\hline 5219 & 239 & & 141 \\
\hline 5305 & 240 & & 78 \\
\hline 3709 & 241 & 16 & \\
\hline 3373 & 242 & 81 & \\
\hline 2429 & 243 & 27 & \\
\hline 2424 & 243 & 28 & \\
\hline 3263 & 245 & 17 & \\
\hline
\end{tabular}

6 Butler D, Ward S, Sweetapple C, Astaraie-Imani M, Diao K, Farmani R., Fu G (2016) Reliable,

7 resilient and sustainable water management: the Safe \& SuRe approach. Glob Chall. DOI:

$8 \quad 10.1002 / \mathrm{gch} 2.1010$.

9 Diao K, Sweetapple C, Farmani R, Fu G, Ward S, Butler D (2016) Global Resilience Analysis

10 of Water Distribution Systems. Water Res 106:383-393. DOI: 10.1016/j.watres.2016.10.011

11 Dijkstra EW (1959) A note on two problems in connexion with graphs. Numerische

12 Mathematik In Numerische Mathematik 1(1):269-271. 
13 Herrera M, Abraham E, Stoianov I (2016) A Graph-Theoretic Framework for Assessing the

14 Resilience of Sectorised Water Distribution Networks. Water Resour Manag 30(6):1685-1699.

15 https://doi.org/10.1007/s11269-016-1245-6

16 Hwang H, Lansey K (2017) Water Distribution System Classification Using System

17 Characteristics and Graph-Theory Metrics. J Water Resour Plan Manag 143(12):1-13.

18 Jacobs P, Goulter IC (1989). Optimization of redundancy in Water Distribution Networks using 19 graph theoretic principles. Eng Optim 15(1):71-82.

20 Meng F, Fu G, Farmani R, Sweetapple C, Butler D (2018) Topological attributes of network

21 resilience: A study in water distribution systems. Water Res 143:376-386

22 Ostfeld A (2005) Water Distribution Systems Connectivity Analysis. J Water Resour Plan 23 Manag 131(1):58-66.

24 Pagano A, Pluchinotta I, Giordano R, Vurro M (2017). Drinking water supply in resilient cities:

25 Notes from L'Aquila earthquake case study. Sustain Cities Soc 28, 435-449. 26 https://doi.org/10.1016/j.scs.2016.09.005

27 Pagano A, Pluchinotta I, Giordano R, Fratino U. (2018a). Integrating "hard" and "soft" 28 infrastructural resilience assessment for water distribution systems. Complex 2018:3074791. https://doi.org/10.1155/2018/3074791

Pagano A, Pluchinotta I, Giordano R, Petrangeli AB, Fratino U, Vurro M (2018b). Dealing 31 with Uncertainty in Decision-Making for Drinking Water Supply Systems Exposed to Extreme 32 Events. Water Resour Manag 32(6), 2131-2145. https://doi.org/10.1007/s11269-018-1922-8

33 Porse E, Lund J (2016) Network Analysis and Visualizations of Water Resources Infrastructure 34 in California: Linking Connectivity and Resilience. J Water Resour Plan Manag 142(1), 4015041. 
36 Torres JM, Duenas-Osorio L, Li Q, Yazdani A (2016) Exploring Topological Effects on Water

37 Distribution System Performance Using Graph Theory and Statistical Models. J Water Resour 38 Plan Manag 4016068-1:1-16.

39 Shin S, Lee S, Judi D, Parvania M, Goharian E, McPherson T, Burian S (2018) A Systematic 40 Review of Quantitative Resilience Measures for Water Infrastructure Systems. Water 10(2), $41 \quad 164$.

42 Walski TM (1993) Water distribution valve topology for reliability analysis. Reliab Eng Syst 43 Saf 42(1):21-27.

44 Yazdani A, Dueñas-Osorio L, Li Q (2013) A scoring mechanism for the rank aggregation of 45 network robustness. Commun Nonlinear Sci Numer Simul 18(10):2722-2732.

46 Yazdani A, Jeffrey P (2012) Water distribution system vulnerability analysis using weighted 47 and directed network models. Water Resour Res 48(6):1-10.

48 Yazdani A, Otoo RA, Jeffrey P (2011) Resilience enhancing expansion strategies for water 49 distribution systems: A network theory approach. Environ Model Softw 26(12):1574-1582.

50 Zeng F, Li X, Li K (2017) Modeling complexity in engineered infrastructure system: Water 51 distribution network as an example. Chaos:27(023105). doi: 10.1063/1.4975762 